\title{
Acknowledgment of Reviewers
}

As we complete the 2018 publication cycle of Psychiatric Rehabilitation Journal, we take this opportunity to acknowledge the valued contributions of our $P R J$ reviewers and thank them for their efforts. With their expertise and support, we published four issues of $P R J$ this year. Our reviewers have graciously provided their time and expert guidance to ensure that authors receive feedback and direction to improve their submissions. Authors in turn, express their appreciation of reviewers' input, and comment that their articles and brief reports are much improved as a result. In addition to acknowledging and thanking our Editorial Review Board, we extend our sincere thanks and gratitude to the individuals listed on this page for their time, expertise, and commitment to conducting reviews for $P R J$ during the time period of October 2017 to October 2018.

Jennifer M. Aakre

Kristen M. Abraham

Donna Ames

Kelly A. Aschbrenner

Tim Daniel Aubry

Amit Baumel

Margherita Bechi

Deborah R. Becker

Dror Ben-Zeev

Victoria Bird

Christina Blomdahl

Crystal R. Blyler

Kelsey A. Bonfils

Wendy Bryant

Jane K. Burke-Miller

Erika Carr

Shu Ping Chen

Amy Neubauer Cohen

Gary Cuddeback

Kristin Davis

Louanne Davis

Paolo del Vecchio

Dominick DePhilippis

Faith B. Dickerson

Amy L. Drapalski

Kimberly Dreison

Johanne Eliacin

Sue E. Estroff

Ruth L. Firmin

\author{
Kathryn Fletcher \\ Rochelle Frounfelker \\ Dan Fulford \\ Gilad Gal \\ Crystal M. Glover \\ Paul B. Gold \\ Jennifer D. Gottlieb \\ Johanna K. P. Greeson \\ Georgina Gross \\ Gretchen L. Haas \\ Joanna Henderson \\ Benjamin Henwood \\ Rochelle Hine \\ Heather Horton \\ Natalie Hundt \\ Marcia G. Hunt \\ Sean A. Kidd \\ Juliet King \\ Toni King \\ Nancy Koroloff \\ Derrick Kranke \\ Marina Kukla \\ Matthew M. Kurtz \\ Jonathon E. Larson \\ Mary Leamy \\ Shannon Lenze \\ Itzhak Levav \\ Rachel Loewy \\ Lauren Luther \\ Paul Lysaker
}

Amanda Lyskawa

Angus MacBeth

James Mandiberg

Marianne Matthias

Bryan McCormick

Alan B. McGuire

Colleen E. McKay

Alice Medalia

Piper Meyer-Kalos

Rebecca Miller

Wan Ming Wai

Kyle S. Minor

Talya Miron-Shatz

Aubrey Moe

Maria Monroe-DeVita

Lisa Mueller

Michelle G. Mullen

John Naslund

Valerie A. Noel

Maria O'Connell

Thomas O'Hare

Jason Peer

Marieke Pijnenborg

Sarah I. Pratt

Michael Pullmann

Bernd Puschner

Andrea Reupert

John Rio

Michael Rowe

\author{
Kathryn Sabella \\ Doreen Salina \\ Jennifer Sánchez \\ Alice Saperstein \\ Mary V. Seeman \\ Steven Paul Segal \\ David Shern \\ Meaghan Stacy \\ Brendon Stubbs \\ Laura Stull \\ Amir Tal \\ Miriam Tepper \\ Jack Tsai
}

Lisa Uebelacker

Lisette van der Meer

Wouter Vanderplasschen

Vanessa Vorhies Klodnick

Melissa SuzanneMaria

Wattenberg

Perla Werner

Sarah Krill Williston

Til Wykes

Scott Yamamoto

Philip T. Yanos

Yaara Zisman-Ilani 